\title{
Guaranteed Convergence Rate for Linear-Quadratic Optimal Time-varying Observers
}

\author{
Mikael Stocks ${ }^{\dagger}$ and Alexander Medvedev ${ }^{\ddagger}$
}

\begin{abstract}
A well-known method by Anderson and Moore for optimal quadratic feedback design with guaranteed convergence rate for linear time-invariant systems is generalized to linear complex-valued time-varying systems and convergence rates. The resulting method is applied to observer design and illustrated by solving the problem of flux estimation in induction machines. A pre-assigned time-varying convergence rate is shown to improve the observer's transients in comparison with a constant one. The suggested design technique can be readily utilized for nonlinear state-affine systems.
\end{abstract}

\section{BACKGROUND}

Consider a linear time-invariant system

$$
\begin{aligned}
\dot{x} & =\mathbf{A} x+\mathbf{B} u \\
y & =\mathbf{C} x
\end{aligned}
$$

where $x \in \mathcal{R}^{n}, u \in \mathcal{R}^{m}, y \in \mathcal{R}^{\ell},(\mathbf{A}, \mathbf{B})$ is a controllable pair and $\mathbf{A}, \mathbf{B}, \mathbf{C}$ are real matrices of suitable dimensions. The problem of optimal quadratic control of (1) with a prescribed degree of stability was first solved in [1] and later presented in book format in [2].

Let $\overline{\mathbf{P}}$ be the stationary solution (i. e. $\overline{\mathbf{P}}=$ $\left.\lim _{t \rightarrow-\infty} \mathbf{P}\left(t, t_{0}\right)\right)$ of the Riccati equation

$$
\begin{aligned}
-\dot{\mathbf{P}} & =\mathbf{P}\left(\mathbf{A}+\frac{1}{2} \alpha \mathbf{I}\right)+\left(\mathbf{A}^{T}+\frac{1}{2} \alpha \mathbf{I}\right) \mathbf{P} \\
& -\mathbf{P B R}^{-1} \mathbf{B}^{T} \mathbf{P}+\mathbf{G G}^{T}
\end{aligned}
$$

with the boundary condition $\mathbf{P}\left(t_{0}, t_{0}\right)$. Then the constant feedback gain control law

$$
u=-\mathbf{R}^{-1} \mathbf{B}^{T} \overline{\mathbf{P}} x
$$

minimizes the quadratic criterium

$$
V(u(\cdot))=\int_{0}^{\infty} e^{\alpha t}\left(u^{T} \mathbf{R} u+x^{T} \mathbf{G G}^{T} x\right) d t
$$

where $\mathbf{R}>0,(\mathbf{G}, \mathbf{A})$ is an observable pair and $\alpha$ is a nonnegative constant. Besides, the closed-loop state vector governed by

$$
\dot{x}=\left(\mathbf{A}-\mathbf{R}^{-1} \mathbf{B}^{T} \overline{\mathbf{P}}\right) x
$$

approaches zero at least as fast as $e^{-\frac{1}{2} \alpha t}$. Since $\alpha$ can be chosen freely and beforehand, the latter property is referred to as prescribed degree of stability.

Generalizing the method of Anderson-Moore to a timevarying case, Linear-Quadratic (LQ) optimality of the design

${ }^{\dagger}$ Systems and Interaction, Luleå University of Technology, SE-971 87 Luleå, SWEDEN, e-mail: Stocks@ltu.se

$\ddagger$ Information Technology, Uppsala University, SE-75105 Uppsala, SWEDEN, e-mail: Alexander.Medvedev@it.uu.se is usually sacrificed for the sake of simplicity. In [3], the stabilization of

$$
\dot{x}=\mathbf{A}(t) x+\mathbf{B}(t) u
$$

with the transition matrix $\mathbf{\Phi}_{\mathbf{A}}\left(t, t_{0}\right)$ being the unique solution to

$$
\dot{\boldsymbol{\Phi}}_{\mathbf{A}}\left(t, t_{0}\right)=\mathbf{A}(t) \mathbf{\Phi}_{\mathbf{A}}\left(t, t_{0}\right) ; \quad \mathbf{\Phi}_{\mathbf{A}}\left(t_{0}, t_{0}\right)=\mathbf{I}
$$

is considered assuming that the controllability gramian

$$
\mathcal{C}_{\mathbf{A}}\left(t_{f}, t_{0}\right)=\int_{t_{0}}^{t_{f}} \boldsymbol{\Phi}_{\mathbf{A}}\left(t_{0}, \theta\right) \mathbf{B}(\theta) \mathbf{B}^{H}(\theta) \boldsymbol{\Phi}_{\mathbf{A}}^{H}\left(t_{0}, \theta\right) d \theta
$$

is non-singular and bounded from above. Then, for any $\alpha>$ 0 , the control law

$$
u(t)=-\beta(t) \mathbf{B}^{H}(t) \mathcal{W}_{\mathbf{A}}^{-1}(t+\tau, t) x(t)
$$

where

$\mathcal{W}_{\mathbf{A}}\left(t_{f}, t_{0}\right)=\int_{t_{0}}^{t_{f}} e^{\alpha\left(t_{0}-\theta\right)} \mathbf{\Phi}_{\mathbf{A}}\left(t_{0}, \theta\right) \mathbf{B}(\theta) \mathbf{B}^{H}(\theta) \boldsymbol{\Phi}_{\mathbf{A}}^{H}\left(t_{0}, \theta\right) d \theta$ and $\beta \in\left[\frac{1}{2}, \infty\right)$ stabilizes (2) with a prescribed degree of stability of at least $\frac{1}{2} \alpha$. Compared to the original method of [1], the controllability conditions are formulated in terms of the original (untransformed) process matrices.

In a dual to the work of [3] approach, the problem of state reconstruction in state-affine systems of the type

$$
\begin{aligned}
\dot{x} & =\mathbf{A}(u) x \\
y & =\mathbf{C} x
\end{aligned}
$$

has been solved in [4] (and followed up in [5]) by the observer

$$
\dot{\hat{x}}=\mathbf{A}(u) \hat{x}-\mathbf{P}^{-1}(t) \mathbf{C}^{T}(\mathbf{C} \hat{x}-y)
$$

where $\mathbf{P}$ is the solution to the Lyapunov equation

$$
-\dot{\mathbf{P}}=\mathbf{P}\left(\mathbf{A}+\frac{1}{2} \alpha \mathbf{I}\right)+\left(\mathbf{A}^{T}+\frac{1}{2} \alpha \mathbf{I}\right) \mathbf{P}-\mathbf{C}^{T} \mathbf{C}
$$

Any vector norm $\|\cdot\|$ of the estimation error $e=\hat{x}-x$ satisfies $\|e(t)\| \leq k e^{-\frac{1}{2} \alpha t}, t \geq 0$ and some constant $k$, whose value depends only on the initial conditions and $u$. It worth to notice here that the actual upper bound provided in [4] relates to the convergence of a Lyapunov function and does not take into account the fact that the estimation error norm converges as a square root of it, thus necessitating the factor $\frac{1}{2}$ in the exponential. The observer exists when, for some $t_{0}, u$ satisfies the "persistency" property

$$
\int_{0}^{t} e^{-\alpha(t-s)} \boldsymbol{\Phi}_{\mathbf{A}}^{T}(s, t) \mathbf{C}^{T} \mathbf{C} \boldsymbol{\Phi}_{\mathbf{A}}(s, t) d s>\mathbf{0}, \quad t \geq t_{0}
$$


The existence condition in (4) involves the desired $\alpha$ and is therefore supposed to be checked for each design.

Summing up, the approaches suggested in [3], [4] achieve guaranteed constant convergence rate in linear time-varying systems. However, some of the design degrees of freedom as well as optimality are lost due to the use of a Lyapunov equation instead of the Riccati equation. Besides, none of the existing design methods can be readily applied to a case of time-varying $\alpha$. The latter is quite useful in applications since the actual convergence rate of a time-varying system is typically also time-varying notwithstanding the stipulated constant upper bound. By enforcing a time-varying guaranteed convergence rate, a more consistent closed-loop behavior of the observer or controller can be obtained.

The contribution of the present paper is the following.

- The optimal design method with prescribed stability rate by Anderson and Moore is generalized to linear complex-valued time-varying systems and convergence rates.

- Existence conditions of the optimal feedback are formulated in terms of the plant properties independent of the desired closed-loop system convergence rate.

- The relationship between the approaches in [1], [2] and in [3], [4], [5] is clarified.

- The resulting design method is illustrated by an application to flux estimation in induction machines.

\section{PROBlem Formulation}

Consider

$$
\begin{aligned}
\dot{x} & =\mathbf{A} x+\mathbf{B} u \\
y & =\mathbf{C} x+\mathbf{D} u
\end{aligned}
$$

where $x \in \mathcal{C}^{n}, u \in \mathcal{C}^{m}, y \in \mathcal{C}^{\ell}$ and matrices $\mathbf{A}, \mathbf{B}, \mathbf{C}$ and $\mathbf{D}$ are time-varying complex-valued matrices of corresponding dimensions. For the sake of brevity, the argument of timevarying matrices and vectors is dropped whenever it does not lead to confusion.

For the purpose of reconstructing $x$ in (5) from the measurements of $u$ and $y$, introduce an observer

$$
\begin{aligned}
\dot{\hat{x}} & =\mathbf{A} \hat{x}+\mathbf{B} u+\mathbf{K}(y-\hat{y}) \\
\hat{y} & =\mathbf{C} \hat{x}+\mathbf{D} u
\end{aligned}
$$

where $\hat{x}$ is an estimate of $x$ and $\mathbf{K}$ is the observer (generally time-varying) gain. Defining the estimation error $e=x-\hat{x}$, the corresponding error system becomes

$$
\dot{e}=(\mathbf{A}-\mathbf{K C}) e
$$

The task is to design $\mathbf{K}$ so that $e \rightarrow 0$ at a given guaranteed convergence rate.

\section{OBSERVER DESIGN}

The observability gramian of (5) on $\mathcal{I}=\left[t_{0}, t_{f}\right]$ is given by

$$
\mathcal{O}_{\mathbf{A}}\left(t_{f}, t_{0}\right)=\int_{t_{0}}^{t_{f}} \boldsymbol{\Phi}_{\mathbf{A}}^{H}\left(\tau, t_{0}\right) \mathbf{C}^{H}(\tau) \mathbf{C}(\tau) \boldsymbol{\Phi}_{\mathbf{A}}\left(\tau, t_{0}\right) d \tau
$$

where ${ }^{H}$ denotes Hermitian adjoint. System (5) (or the pair $(\mathbf{C}, \mathbf{A}))$ is said to be uniformly observable on $\mathcal{I}$ if there is a constant $\delta_{o}>0$ such that the two following conditions hold

$$
\begin{array}{cc}
\alpha_{l} \mathbf{I} \leq \mathcal{O}_{\mathbf{A}}\left(T, T+\delta_{o}\right) \leq \alpha_{u} \mathbf{I}, & t_{0} \leq T \leq t_{f}-\delta_{o} \\
\left\|\boldsymbol{\Phi}_{\mathbf{A}}(t, \tau)\right\|_{p} \leq f(|t-\tau|), & t, \tau \in \mathcal{I}
\end{array}
$$

for some $\alpha_{l}, \alpha_{u}>0, f(\cdot): \mathcal{R} \rightarrow \mathcal{R}$ is bounded on bounded intervals, and $\|\cdot\|_{p}$ is the induced matrix $p$-norm.

Uniform controllability of system (5) (or of the pair $(\mathbf{A}, \mathbf{B}))$ is defined dually to observability considering the controllability gramian

$$
\mathcal{C}_{\mathbf{A}}\left(t_{f}, t_{0}\right)=\int_{t_{0}}^{t_{f}} \boldsymbol{\Phi}_{\mathbf{A}}\left(t_{0}, \tau\right) \mathbf{B}(\tau) \mathbf{B}^{H}(\tau) \boldsymbol{\Phi}_{\mathbf{A}}^{H}\left(t_{0}, \tau\right) d \tau
$$

Lemma 1: Let $\alpha(t)$ be a function $\mathcal{R} \rightarrow \mathcal{R}$ and $|\alpha(t)| \leq$ $\alpha_{\max }, t \in \mathcal{I}$, where $\alpha_{\max }$ is a constant. Then $\left(\mathbf{C}, \mathbf{A}+\frac{1}{2} \alpha \mathbf{I}\right)$ $\left(\left(\mathbf{A}+\frac{1}{2} \alpha \mathbf{I}, \mathbf{B}\right)\right)$ is uniformly observable (controllable) pair one some non-trivial finite or infinite $\mathcal{I}$ if and only if $(\mathbf{C}, \mathbf{A})$ $((\mathbf{A}, \mathbf{B}))$ is a uniformly observable (controllable) pair on the same interval.

Proof: The proof is given only for the observability case taking into account the duality of that for controllability. Define

$$
\mathbf{E}\left(t, t_{0}\right)=\boldsymbol{\Phi}_{\mathbf{A}+\frac{1}{2} \alpha \mathbf{I}}\left(t, t_{0}\right)-\boldsymbol{\Phi}_{\mathbf{A}}\left(t, t_{0}\right) \boldsymbol{\Phi}_{\frac{1}{2} \alpha \mathbf{I}}\left(t, t_{0}\right)
$$

By differentiating, using properties of transition matrices and reordering terms one obtains

$$
\dot{\mathbf{E}}\left(t, t_{0}\right)=\left(\mathbf{A}(t)+\frac{1}{2} \alpha(t) \mathbf{I}\right) \mathbf{E}\left(t, t_{0}\right) ; \quad \mathbf{E}\left(t_{0}, t_{0}\right)=\mathbf{0}
$$

hence $\mathbf{E}=\mathbf{0}, \forall t$. Now note that

$$
\mathbf{\Phi}_{\frac{1}{2} \alpha \mathbf{I}}\left(t, t_{0}\right)=e^{\frac{1}{2} \gamma\left(t, t_{0}\right)} \mathbf{I} ; \quad \gamma\left(t, t_{0}\right)=\int_{t_{0}}^{t} \alpha(\tau) d \tau
$$

and thus $\boldsymbol{\Phi}_{\frac{1}{2} \alpha \mathbf{I}}\left(t, t_{0}\right)$ commutes with any compatible matrix. Therefore $\boldsymbol{\Phi}_{\mathbf{A}} \boldsymbol{\Phi}_{\frac{1}{2} \alpha \mathbf{I}}=\boldsymbol{\Phi}_{\frac{1}{2} \alpha \mathbf{I}} \boldsymbol{\Phi}_{\mathbf{A}}$ and

$$
\begin{aligned}
\boldsymbol{\Phi}_{\mathbf{A}+\frac{1}{2} \alpha \mathbf{I}} & =\boldsymbol{\Phi}_{\mathbf{A}} \boldsymbol{\Phi}_{\frac{1}{2} \alpha \mathbf{I}}=\boldsymbol{\Phi}_{\frac{1}{2} \alpha \mathbf{I}} \boldsymbol{\Phi}_{\mathbf{A}} \\
\boldsymbol{\Phi}_{\mathbf{A}+\frac{1}{2} \alpha \mathbf{I}}\left(t, t_{0}\right) & =e^{\frac{1}{2} \gamma\left(t, t_{0}\right)} \boldsymbol{\Phi}_{\mathbf{A}}\left(t, t_{0}\right)
\end{aligned}
$$

Since

$$
0<e^{\gamma\left(\tau, t_{0}\right)} \leq e^{\alpha_{\max }\left(\tau-t_{0}\right)}, \quad t_{0} \leq \tau
$$

equation (9) implies that condition (8) holds for $\boldsymbol{\Phi}_{\mathbf{A}+\frac{1}{2} \alpha \mathbf{I}}$ whenever it does for $\mathbf{\Phi}_{\mathbf{A}}$ and vice versa.

Utilizing (9), the observability gramian $\mathcal{O}_{\mathbf{A}+\frac{1}{2} \alpha \mathbf{I}}\left(t_{f}, t_{0}\right)$ is evaluated to

$\mathcal{O}_{\mathbf{A}+\frac{1}{2} \alpha \mathbf{I}}=\int_{t_{0}}^{t_{f}} e^{\gamma\left(\tau, t_{0}\right)} \mathbf{\Phi}_{\mathbf{A}}^{H}\left(\tau, t_{0}\right) \mathbf{C}^{H}(\tau) \mathbf{C}(\tau) \mathbf{\Phi}_{\mathbf{A}}\left(\tau, t_{0}\right) d \tau$

Recalling (10) and the fact that $e^{\gamma\left(\tau, t_{0}\right)}$ is continuous in $\tau$ enables the use of the Generalized mean value theorem of integral calculus for some point $\xi \in\left[t_{0}, t_{f}\right]$

$$
\mathcal{O}_{\mathbf{A}+\frac{1}{2} \alpha \mathbf{I}}\left(t_{f}, t_{0}\right)=e^{\gamma\left(\xi, t_{0}\right)} \mathcal{O}_{\mathbf{A}}\left(t_{f}, t_{0}\right)
$$

Since $e^{\gamma\left(\xi, t_{0}\right)}$ is a scalar constant, condition (7) holds for $\mathcal{O}_{\mathbf{A}+\frac{1}{2} \alpha \mathbf{I}}$ whenever it holds for $\mathcal{O}_{\mathbf{A}}$. Thus, it is proven that 
both (7) and (8) equivalently apply to $\mathcal{O}_{\mathbf{A}+\frac{1}{2} \alpha \mathbf{I}}, \boldsymbol{\Phi}_{\mathbf{A}+\frac{1}{2} \alpha \mathbf{I}}$ as well as to $\mathcal{O}_{\mathbf{A}}, \boldsymbol{\Phi}_{\mathbf{A}}$ and the observability result follows. The result for controllability is obtained similarly.

Remark 1: For the time-invariant case, the result of Lemma 1 follows immediately from Lemma 3 in [6] by observing that the matrix function $s+\left.\alpha\right|_{s=\mathbf{A}}$ does not change the Jordan structure of A. See also Problem 3.5-5 in [2].

Remark 2: The results of Lemma 1 also hold for $\alpha(t) \in$ $\mathcal{C}$, which can be verified by slightly modifying the proof.

Let the feedback matrix $\mathbf{K}$ be given by

$$
\begin{aligned}
\mathbf{K} & =\mathbf{P} \mathbf{C}^{H} \mathbf{Q} \\
\dot{\mathbf{P}} & =\alpha \mathbf{P}+\mathbf{A} \mathbf{P}+\mathbf{P A}{ }^{H}-\mathbf{P} \mathbf{C}^{H} \mathbf{Q C P}+\mathbf{G G}^{H}
\end{aligned}
$$

where $\mathbf{P}\left(t_{0}\right)=\mathbf{P}_{\mathbf{0}}, \mathbf{Q}$ is chosen so that

$$
\mathbf{C}^{H} \mathbf{Q C} \triangleq \mathbf{H}^{H} \mathbf{H} \geq \mathbf{0}
$$

and $\mathbf{G}$ is a time-varying matrix of suitable dimensions. The estimation error of the observer is then governed by

$$
\dot{e}=\left(\mathbf{A}-\mathbf{P} \mathbf{H}^{H} \mathbf{H}\right) e
$$

Theorem 1 (Bounded positive definite solution): Assume that for some constant $\alpha_{\max }$

$$
|\alpha(t)| \leq \alpha_{\max }, \quad \alpha(t) \in \mathcal{R}, t \in \mathcal{I}
$$

$(\mathbf{H}, \mathbf{A})$ is an uniformly observable pair on $\mathcal{I}$ and $\mathbf{P}_{\mathbf{0}}>\mathbf{0}$. In addition, let at least one of the following alternatives hold:

I: $\quad(\mathbf{A}, \mathbf{G})$ is a uniformly controllable pair on $\mathcal{I}$

II: the system $\dot{\xi}=\left(\mathbf{A}+\frac{1}{2} \alpha \mathbf{I}\right) \xi$ is diverging so that

$$
\left\|\boldsymbol{\Phi}_{\mathbf{A}+\frac{1}{2} \alpha \mathbf{I}}\left(t, t_{0}\right)\right\|_{p} \geq K e^{\beta\left(t-t_{0}\right)}, \quad K, \beta>0
$$

Then

$$
c_{l} \mathbf{I} \leq \mathbf{P}(t) \leq c_{u} \mathbf{I}, \quad t \in \mathcal{I}
$$

for some $c_{l}, c_{u}>0$.

Proof: First notice that (11) can be written as

$\dot{\mathbf{P}}=\left(\mathbf{A}+\frac{1}{2} \alpha \mathbf{I}\right) \mathbf{P}+\mathbf{P}\left(\mathbf{A}+\frac{1}{2} \alpha \mathbf{I}\right)^{H}-\mathbf{P} \mathbf{H}^{H} \mathbf{H} \mathbf{P}+\mathbf{G G}^{H}$

I: According to Theorem 2.1 in [7], all the solutions to the Riccati equation above satisfy (14) if $\left(\mathbf{H}, \mathbf{A}+\frac{1}{2} \alpha \mathbf{I}\right)$ is uniformly observable and $\left(\mathbf{A}+\frac{1}{2} \alpha \mathbf{I}, \mathbf{G}\right)$ is uniformly controllable. Lemma 1 proves that $\left(\mathbf{H}, \mathbf{A}+\frac{1}{2} \alpha \mathbf{I}\right)\left(\left(\mathbf{A}+\frac{1}{2} \alpha \mathbf{I}, \mathbf{G}\right)\right)$ being uniformly observable (controllable) is equivalent to uniform observability (controllability) of $(\mathbf{H}, \mathbf{A})((\mathbf{A}, \mathbf{G}))$ which implies upper and lower boundness of $\mathbf{P}(t)>\mathbf{0}$.

II: Let $\tilde{\mathbf{P}}$ denote the solution of (11) for $\mathbf{G}=\mathbf{0}$. Written in terms of $\tilde{\mathbf{P}}^{-1}$, (11) takes the form

$$
-\frac{d}{d t} \tilde{\mathbf{P}}^{-1}=\tilde{\mathbf{P}}^{-1}\left(\mathbf{A}+\frac{1}{2} \alpha \mathbf{I}\right)+\left(\mathbf{A}+\frac{1}{2} \alpha \mathbf{I}\right)^{H} \tilde{\mathbf{P}}^{-1}-\mathbf{H}^{H} \mathbf{H}
$$

The Lyapunov equation above has exactly the same structure as equation (3). For a uniformly observable pair $(\mathbf{H}, \mathbf{A}+$ $\left.\frac{1}{2} \alpha \mathbf{I}\right)$ or, equivalently, a uniformly observable pair $(\mathbf{H}, \mathbf{A})$ (by applying Lemma 1), Lemma 2.1 in [5] guarantees that
$\tilde{\mathbf{P}}^{-1}$ is positive definite as well as lower and upper bounded. Therefore

$$
c_{l} \mathbf{I} \leq \tilde{\mathbf{P}}(t) \leq c_{u}^{\prime} \mathbf{I}
$$

for some $c_{l}, c_{u}^{\prime}>0$. Notice here that for $\alpha(t) \equiv 0$, matrix differential equation (15) converges for matrices A leading to diverging dynamics (in the sense of (13)) and diverges for matrices $\mathbf{A}$ leading to converging ones. Therefore, condition (13) safeguards the boundness of $\tilde{\mathbf{P}}$.

Now, consider the solution to (11) for an arbitrary $\mathbf{G}$. The matrix $\mathbf{R}=\mathbf{P}-\tilde{\mathbf{P}}$ satisfies

$$
\dot{\mathbf{R}}=\overline{\mathbf{A}} \mathbf{R}+\mathbf{R} \overline{\mathbf{A}}^{H}+\mathbf{G G}^{H} ; \quad \mathbf{R}\left(t_{0}\right)=\mathbf{0}
$$

where $\overline{\mathbf{A}}=\mathbf{A}+\frac{1}{2} \alpha \mathbf{I}-\mathbf{H}^{H} \mathbf{H} \tilde{\mathbf{P}}-\frac{1}{2} \mathbf{H}^{H} \mathbf{H R}$. Integrating (16) gives

$$
\mathbf{R}(t)=\int_{t_{0}}^{t} \boldsymbol{\Phi}_{\overline{\mathbf{A}}}(t, \tau) \mathbf{G}(\tau) \mathbf{G}^{H}(\tau) \boldsymbol{\Phi}_{\overline{\mathbf{A}}}^{H}(t, \tau) d \tau \geq \mathbf{0}
$$

Therefore $\mathbf{P}=\mathbf{R}+\tilde{\mathbf{P}} \geq \tilde{\mathbf{P}} \geq c_{l} \mathbf{I}$. The fact that $\mathbf{P}$ is as well upper bounded can be concluded from Lemma 2.1 in [7] observing that the upper bound on the solutions of the Riccati equation provided in it holds without assuming controllability of $(\mathbf{A}, \mathbf{G})$. Therefore $\mathbf{P} \leq c_{u} \mathbf{I}$, where $c_{u} \geq$ $c_{u}^{\prime}$. Finally, Lemma 1 proves that $\left(\mathbf{H}, \mathbf{A}+\frac{1}{2} \alpha \mathbf{I}\right)$ being uniformly observable is equivalent to uniform observability of $(\mathbf{H}, \mathbf{A})$, hence upper and lower boundness of $\mathbf{P}(t)>\mathbf{0}$ follows for alternative II.

Remark 3: Notice that the solution to Riccati equation (11) continuously depends on $\alpha$ for $\mathbf{P}\left(t_{0}\right) \geq \mathbf{0}$. Let $\mathbf{P}_{1}$ and $\mathbf{P}_{2}$ satisfy

$$
\begin{aligned}
\dot{\mathbf{P}}_{1} & =\left(\mathbf{A}+\frac{1}{2} \alpha_{1} \mathbf{I}\right) \mathbf{P}_{1}+\mathbf{P}_{1}\left(\mathbf{A}+\frac{1}{2} \alpha_{1} \mathbf{I}\right)^{H} \\
& -\mathbf{P}_{1} \mathbf{H}^{H} \mathbf{H} \mathbf{P}_{1}+\mathbf{G G}^{H} \\
\dot{\mathbf{P}}_{2} & =\left(\mathbf{A}+\frac{1}{2} \alpha_{2} \mathbf{I}\right) \mathbf{P}_{2}+\mathbf{P}_{2}\left(\mathbf{A}+\frac{1}{2} \alpha_{2} \mathbf{I}\right)^{H} \\
& -\mathbf{P}_{2} \mathbf{H}^{H} \mathbf{H} \mathbf{P}_{2}+\mathbf{G G}^{H}
\end{aligned}
$$

for some $\alpha_{1}(t) \leq \alpha_{2}(t), t \in \mathcal{I}$ and $\mathbf{P}_{1}\left(t_{0}\right)=\mathbf{P}_{2}\left(t_{0}\right) \geq \mathbf{0}$. Denote $\Delta \mathbf{P}(t)=\mathbf{P}_{2}(t)-\mathbf{P}_{1}(t)$. The evolution of $\Delta \mathbf{P}$ is given by

$$
\begin{aligned}
\Delta \dot{\mathbf{P}} & =\alpha_{2} \Delta \mathbf{P} \\
& +\left(\mathbf{A}-\mathbf{P}_{1} \mathbf{H}^{H} \mathbf{H}\right) \Delta \mathbf{P}+\Delta \mathbf{P}\left(\mathbf{A}-\mathbf{P}_{1} \mathbf{H}^{H} \mathbf{H}\right)^{H} \\
& -\Delta \mathbf{P H} \mathbf{H}^{H} \mathbf{H} \Delta \mathbf{P}+\left(\alpha_{2}-\alpha_{1}\right) \mathbf{P}_{1} ; \quad \Delta \mathbf{P}\left(t_{0}\right)=\mathbf{0}
\end{aligned}
$$

Being initialized at $\Delta \mathbf{P}\left(t_{0}\right)=\mathbf{0}$, the Riccati equation above maintains $\Delta \mathbf{P}(t) \geq \mathbf{0}$. Thus $\alpha_{2}-\alpha_{1} \geq 0, t \in \mathcal{I}$ implies $\mathbf{P}_{2}(t)-\mathbf{P}_{1}(t) \geq \mathbf{0}, t \in \mathcal{I}$.

Theorem 2 (Exponential Convergence): Assume that the conditions of Theorem 1 hold and $\alpha(t) \geq \alpha_{\min } \geq 0, t \in$ $\mathcal{I}$ for some constant $\alpha_{\min }$. Then a solution to (12) converges exponentially with a rate of

$$
\|e(t)\| \leq \lambda^{\prime} e^{-\frac{1}{2} \gamma\left(t, t_{0}\right)} \leq \lambda e^{-\frac{1}{2} \alpha_{\min }\left(t-t_{0}\right)}, t \in \mathcal{I}
$$

for some $\lambda^{\prime}, \lambda \geq 0$. 
Proof: Consider the Lyapunov function candidate $V=$ $e^{H} \mathbf{P}^{-1} e$ and its derivative

$$
\dot{V}=-\alpha V-e^{H}\left(\mathbf{H}^{H} \mathbf{H}+\mathbf{P}^{-1} \mathbf{G G}^{H} \mathbf{P}^{-1}\right) e
$$

By integrating the equation above and taking into account the boundness (due to Theorem 1) and symmetricity of $\mathbf{P}$, it follows that

$$
\begin{gathered}
V(t) \leq e^{-\gamma\left(t, t_{0}\right)} V\left(t_{0}\right) \leq e^{-\alpha_{\min }\left(t-t_{0}\right)} V\left(t_{0}\right) \quad \Rightarrow \\
\|e(t)\| \leq \lambda^{\prime} e^{-\frac{1}{2} \gamma\left(t, t_{0}\right)} \leq \lambda e^{-\frac{1}{2} \alpha_{\min }\left(t-t_{0}\right)}
\end{gathered}
$$

for some $\lambda^{\prime}, \lambda \geq 0$.

Remark 4: In practice, Alternative I of Theorem 1 can always utilized to achieve stability and convergence if $(\mathbf{H}, \mathbf{A})$ is a uniformly observable pair, since $\mathbf{G}$ is a design degree of freedom. On the other hand, Alternative II allows for the choice $\mathbf{G}=\mathbf{0}$. However, care must be taken to select $\alpha$ ensuring the "instability" ( $c f(13)$ ) of $\mathbf{A}+\frac{1}{2} \alpha \mathbf{I}$. This basically reduces the approach of Alternative II in Theorem 1 to the case treated in [4], [5] or, dually, in [3] for the controller design.

Remark 5: The transformation approach suggested for LTI systems in [2] is still valid in the time-varying case. Introduce

$$
\begin{aligned}
& x_{\alpha}(t)=e^{\frac{1}{2} \gamma\left(t, t_{0}\right)} x(t) \\
& u_{\alpha}(t)=e^{\frac{1}{2} \gamma\left(t, t_{0}\right)} u(t)
\end{aligned}
$$

The state equation of (5) reads then in terms of the new variables as

$$
\dot{x}_{\alpha}=\left(\mathbf{A}+\frac{1}{2} \alpha \mathbf{I}\right) x_{\alpha}+\mathbf{B} u_{\alpha}
$$

and (17) precisely gives the relationship between the convergence of $x$ and that of $x_{\alpha}$. This actually paves the way to a simple alternative proof for the observer convergence rate corresponding to Alternative I in Theorem 1. Preservation of controllability and observability under the transformation should yet be checked independently. Neither Alternative II can be treated in this framework. Therefore, the approach undertaken in the present paper is unifying for the original work by Anderson-More and the later contributions in the field of state-affine systems.

Remark 6: Since Riccati equation (11) is solved for evaluating the feedback gain $\mathbf{K}$ in (6), the design is optimal in the sense of minimizing the criterion

$$
\begin{aligned}
U\left(t, t_{0}\right) & =e^{-\gamma\left(t, t_{0}\right)} e^{H}\left(t_{0}\right) \mathbf{P}_{\mathbf{0}} e\left(t_{0}\right) \\
& +\int_{t_{0}}^{t} e^{-\gamma\left(\tau, t_{0}\right)} e^{H}(\tau)\left(\mathbf{C}^{H} \mathbf{Q}^{-1} \mathbf{C}+\mathbf{G G}^{H}\right) e(\tau) d \tau
\end{aligned}
$$

The matrices $\mathbf{Q}, \mathbf{G}, \mathbf{P}_{\mathbf{0}}$ and the function $\alpha(t)$ represent the design degrees of freedom.

\section{Simulation eXAmple}

A simulation example is provided in this section to support the theoretical results above. The problem of magnetic flux estimation in the induction machine (IM) is chosen to demonstrate a technical application of time-varying complexvalued linear models. However, the example is not intended as a perfect solution to the underlying industrial problem but just as a pure illustration with some technical relevance. For instance, no comparison with the numerous alternative flux observers is given in this brief paper. The mathematical model used in the sequel is developed to achieve a parsimonious description of the motor dynamics and all necessary details about it can be found in [8]. Generalized models for faulty IMs, concisely presented, can be found in [9] and [10].

The electrical part of the IM is linear, time-varying and described by

$$
\begin{aligned}
& {\left[\begin{array}{c}
\dot{\bar{\psi}}_{c S} \\
\overline{\bar{x}}_{c S}
\end{array}\right]=\left[\begin{array}{cc}
-\alpha_{S} & \alpha_{S}(1-\mu) \\
\alpha_{r} & -\alpha_{r}+\jmath \bar{\omega}(t)
\end{array}\right]\left[\begin{array}{c}
\bar{\psi}_{c S} \\
\bar{x}_{c S}
\end{array}\right]+\left[\begin{array}{l}
1 \\
0
\end{array}\right] \bar{u}_{c S}} \\
& \imath_{c S}=\frac{K_{S}}{\mu}\left[\begin{array}{ll}
1 & -(1-\mu)
\end{array}\right]\left[\begin{array}{l}
\bar{\psi}_{c S} \\
\bar{x}_{c S}
\end{array}\right]
\end{aligned}
$$

where $\bar{u}_{c S}$ is the normalized stator voltage, $\bar{\psi}_{c S}$ is the normalized stator flux, $\bar{x}_{c S}$ is the normalized stator pseudo flux and $\imath_{c S}$ is the stator current. The parameters for the electrical part correspond to those of a certain real-life general-purpose IM and amount to $K_{S}=133.1, \mu=0.08726, \alpha_{S}=191.0$ and $\alpha_{r}=95.42$. To complete the model, an equation for the mechanical part is needed as well,

$$
\dot{\bar{\omega}}=-\alpha_{m} \bar{\omega}+\delta\left[\jmath \frac{1}{2} \frac{1-\mu}{\mu}\left(\bar{\psi}_{c S}^{H} \bar{x}_{c S}-\bar{\psi}_{c S} \bar{x}_{c S}^{H}\right)-\bar{M}_{l}\right]
$$

where $\bar{\omega}$ is the electrical rotor speed and $\bar{M}_{l}$ is the normalized load on the IM. The parameters of the mechanical part are $\alpha_{m}=0.1, \delta=4 \cdot 10^{6}$.

Throughout the simulations, it is assumed that $\bar{\omega}$ is measured, hence only the state variables of the electrical part are to be estimated. The mechanical equation is used to generate suitable values of $\bar{\omega}$ and is not part of the estimation problem. The simulations have been performed to imitate a start-up of the IM, i.e. with the initial conditions $\bar{\psi}_{c S}(0)=\bar{x}_{c S}(0)=0$ and $\bar{\omega}(0)=0$, since the time-varying dynamics of the motor are most prominent in this mode. The IM is also supposed to be driven by a symmetrical normalized voltage source with a frequency of $50 \mathrm{~Hz}$, i.e. $\bar{u}_{c S}=e^{\jmath 100 \pi t}$, and run without mechanical load $\bar{M}_{l}=0$.

To estimate the state vector of the electrical part of the IM

$$
x=\left[\begin{array}{ll}
\bar{\psi}_{c S} & \bar{x}_{c S}
\end{array}\right]^{T}
$$

observer (6) is employed. In Riccati equation (11) integrated together with the observer and yielding the feedback gain $\mathbf{K}$, the following parameters are used. To begin with, the guaranteed convergence rate of the Lyapunov function is kept constant $\alpha=100$. The design matrices are given as $\mathbf{G}=\mathbf{0}$, $\mathbf{Q}=1$ and $\mathbf{P}(0)=\mathbf{I}$. The design is thus based on the Lyapunov equation.

The initial values of the state estimates are randomly selected as

$$
\begin{aligned}
& \hat{\bar{\psi}}_{c S}(0)=-5.617-\jmath 0.950 \\
& \hat{\bar{x}}_{c S}(0)=-2.829+\jmath 7.716
\end{aligned}
$$


implying $\|e(0)\|_{2}=10$, where $\|\cdot\|_{2}$ denotes euclidean norm. The evolution of the euclidean norm of the observer's estimation error is depicted in Fig. 1. Note that $\|e\|_{2} \leq 10 e^{-\frac{1}{2} \alpha t}=10 e^{-50 t}$. To emphasize the effect of the Riccati-based time-varying feedback, the convergence of the estimation error with the observer running without output estimation error feedback (i.e. $\mathbf{K}=0)\left\|e_{o l}\right\|_{2}$ is also shown in the same figure. In Fig. 2, the maximum and minimum singular values $(\bar{\sigma}\{\mathbf{P}\})$ and $(\underline{\sigma}\{\mathcal{O}\})$, respectively, are depicted. Clearly, the solution to the Riccati equation stays bounded and the motor is observable, the latter is also predicted in [11]. However, at the lower angular velocities in the beginning of the simulation the observability of the electrical part of the IM is yet quite poor.

In Fig. 1, a drastic decrease in the estimation error is seen in the very beginning of the plot. Such high frequency transients are undesirable in motor control where flux observers are broadly employed. This calls for a time-varying $\alpha$ that allows for a smoother and more consistent behavior of the flux estimate, see Fig. 3. There

$$
\alpha(t)=\alpha_{0}\left(1-\mathrm{e}^{-\frac{1}{T_{\alpha}} t}\right) \Rightarrow e^{-\frac{1}{2} \gamma\left(t, t_{0}\right)}=e^{-\frac{1}{2}\left(\alpha_{0} t-\alpha(t) T_{\alpha}\right)}
$$

where $\alpha_{0}=1000, T_{\alpha}=10^{-2}$ and $\mathbf{P}(0)=10^{-5} \mathbf{I}$.

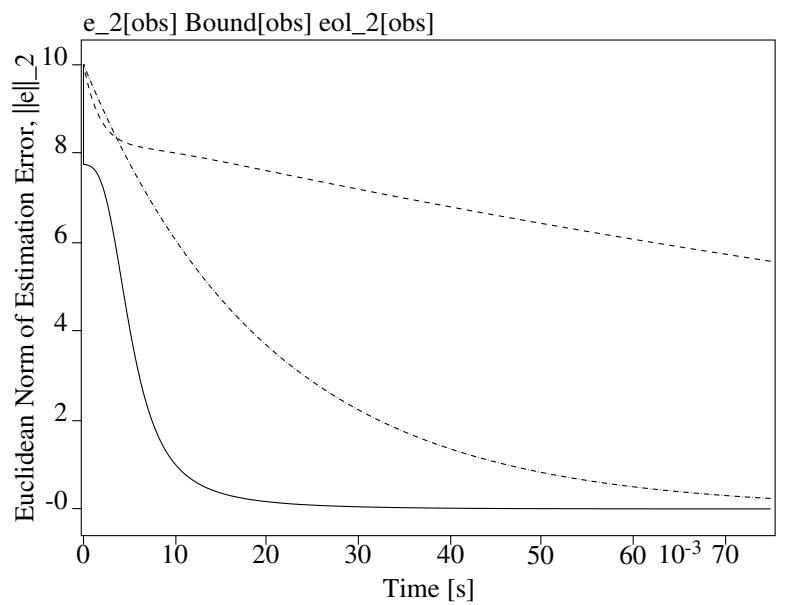

Fig. 1. Convergence of the estimation error. Solid line - Euclidean norm of the estimation error. Dash-dotted line - Upper bound. Dashed line Euclidean norm of the estimation error for the open-loop observer with $\mathbf{K}=0$.

A simulation with a significantly increased value of the matrix $\mathbf{Q}$ is depicted in Fig. 4 where $\alpha=100, \mathbf{Q}=100$, $\mathbf{G}=\mathbf{0}$ and $\mathbf{P}(0)=\mathbf{I}$. Higher values of $\mathbf{Q}$ lead to faster convergence of the estimation error, also compared to the upper bound given by $\alpha$.

In most cases, the bound based on the guaranteed convergence rate is on the conservative side. An example where a good agreement between the upper bound and actual performance is observed can be seen in Fig. 5. The following design parameters are used $\alpha=100, \mathbf{Q}=1, \mathbf{G}=10^{-3} \mathbf{C}^{H}$ and $\mathbf{P}(0)=\mathbf{I}$.

The initial condition to the Riccati equation is also an important design degree of freedom since it influences the
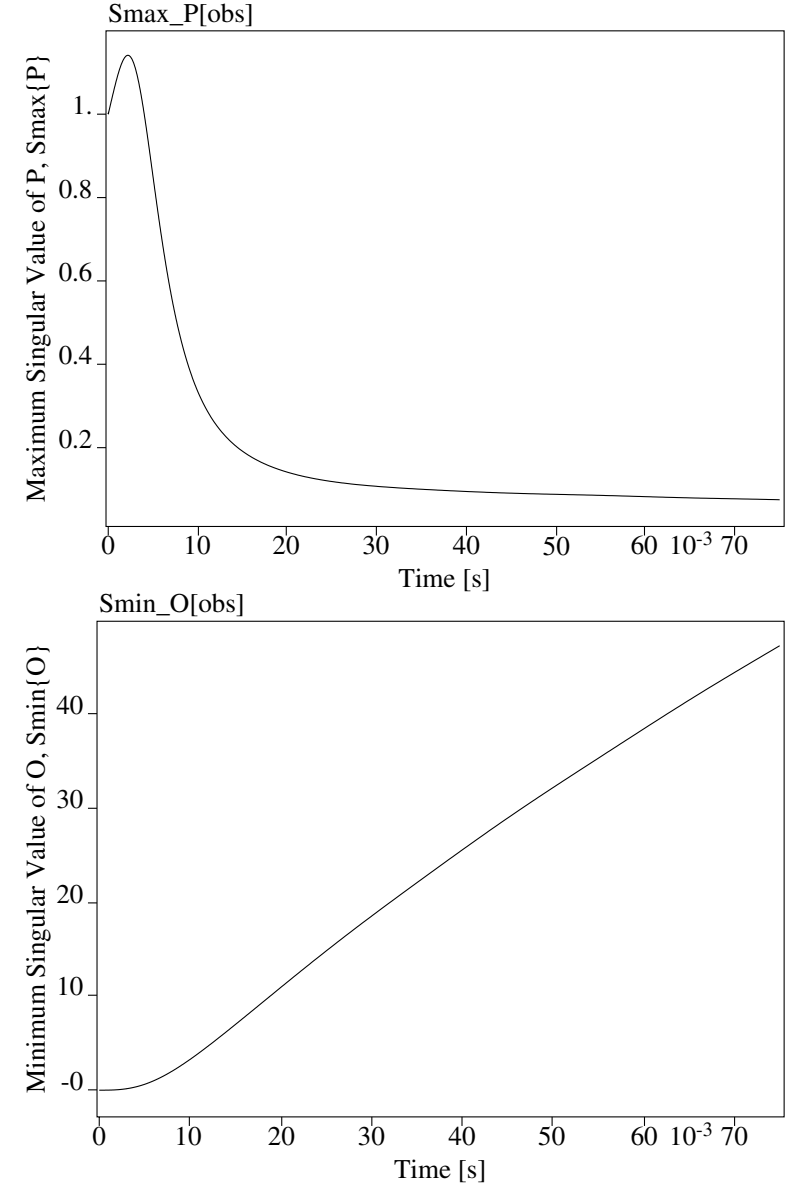

Fig. 2. Singular values. Upper plot $-\bar{\sigma}\{\mathbf{P}\}$. Lower plot $-\underline{\sigma}\{\mathcal{O}\}$.

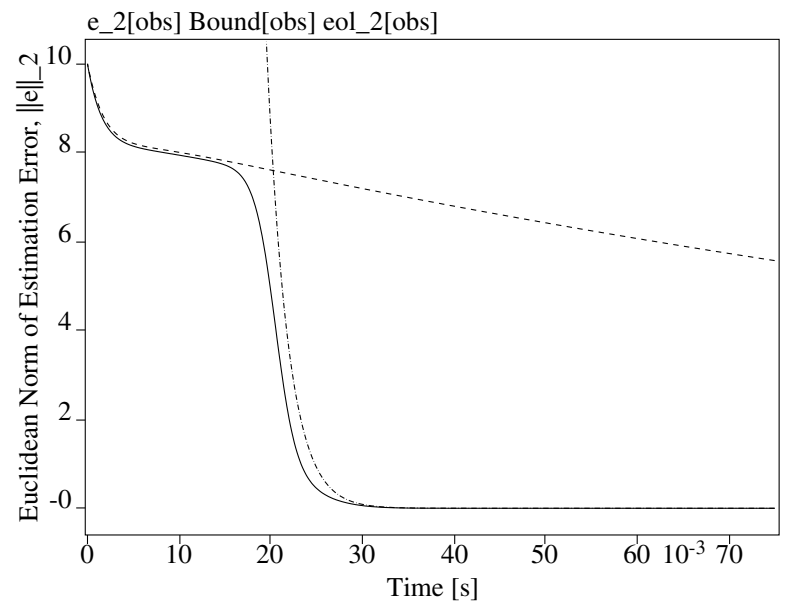

Fig. 3. Convergence of the estimation error with a time-varying $\alpha$. Solid line - Euclidean norm of the estimation error. Dashed line - Euclidean norm of the estimation error for the open-loop observer with $\mathbf{K}=0$. Dash-dotted line - Upper bound. 


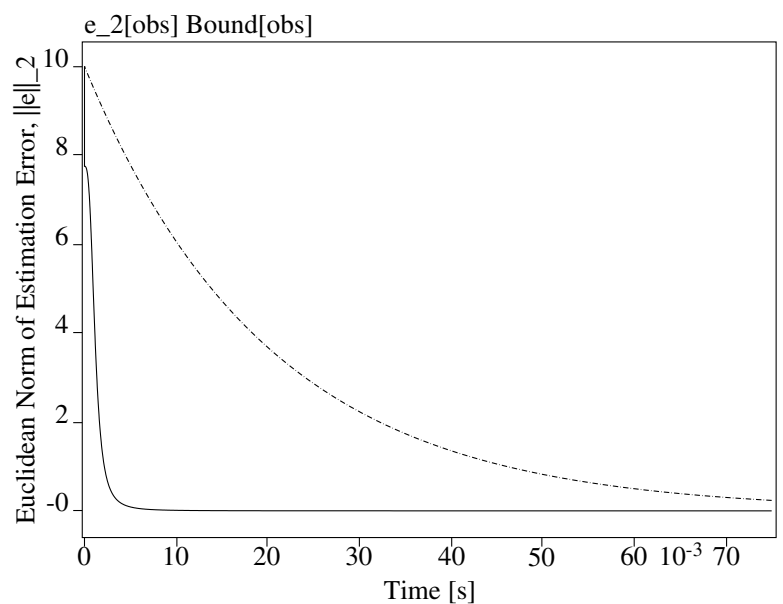

Fig. 4. Convergence of estimation error for $\mathbf{Q}=100, \mathbf{G}=\mathbf{0}$. Solid line - Estimation error. Dash-dotted line - Upper bound.

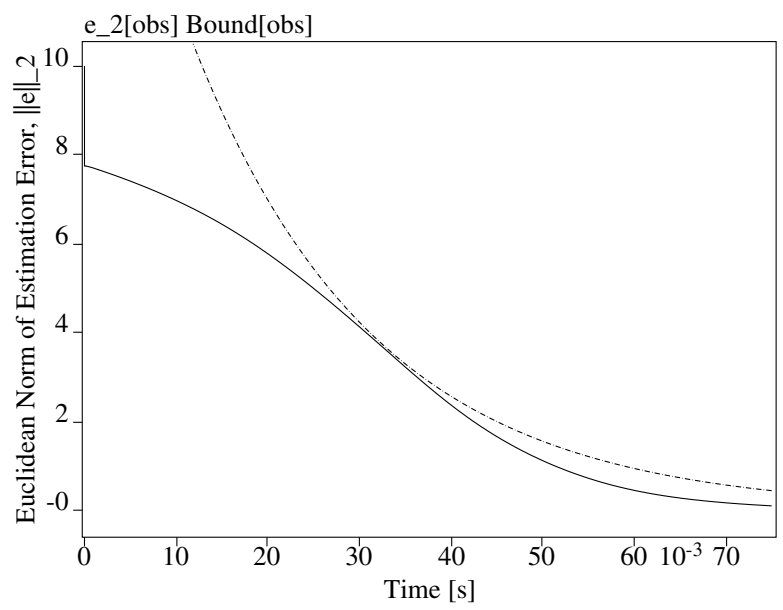

Fig. 5. Tightness illustration for the bound on convergence of estimation error, $\alpha=100, \mathbf{Q}=1, \mathbf{G}=10^{-3} \mathbf{C}^{H}$ and $\mathbf{P}(0)=\mathbf{I}$. Solid line Estimation error. Dash-dotted line - Upper bound.

optimization criterium. A case of an increased value of $\mathbf{P}(0)=10 \mathbf{I}$ is simulated, Fig. 6. Other design parameters are $\alpha=100, \mathbf{Q}=1, \mathbf{G}=10^{-3} \mathbf{C}^{H}$.

\section{CONCLUSiONS}

The classical design method for achieving guaranteed convergence rate in solving optimal linear-quadratic problems suggested by Anderson and Moore is generalized to timevarying systems and convergence rates in application to observer synthesis. Existence conditions of the design methods are formulated in terms of the original plant parameters. Connections of the developed technique with gramian-based controller and observer design methods are pointed out. The available degrees of freedom are illustrated by simulation with respect to the magnetic flux estimation problem in the induction machines.

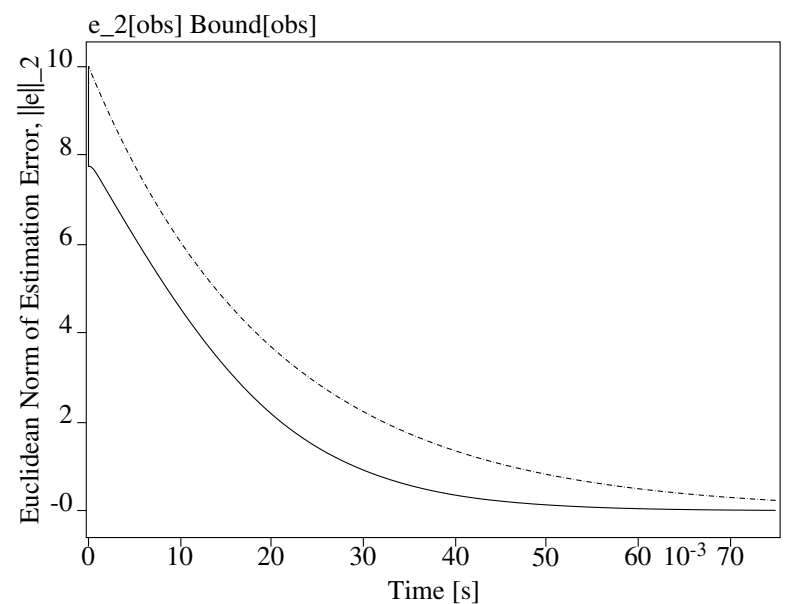

Fig. 6. Convergence of estimation error with $\mathbf{P}(0)=10 \mathbf{I}$. Solid line Estimation error. Dash-dotted line - Upper bound.

\section{REFERENCES}

[1] B. D. O. Andersson and J. B. Moore, "Linear system optimization with prescribed degree of stability," Proc. IEE, vol. 16, no. 12, pp. 2083-2087, December 1969.

[2] - Optimal Control: Linear Quadratic Methods. Prentice-Hall International Editions, 1989.

[3] V. H. L. Cheng, "A direct way to stabilize continuous-time and discrete-time linear time-varying systems," IEEE Transactions on Automatic Control, vol. 24, pp. 641-643, Aug. 1979.

[4] H. Hammouri and J. De Leon Morales, "Observer synthesis for stateaffine systems," in Proc. of the 29th Conference on Decision and Control, Honolulu, Hawaii, 1990.

[5] G. Besancon, G. Bornard, and H. Hammouri, "Observer synthesis for a class of nonlinear control systems," European Journal of Control, vol. 2, 1996.

[6] A. Medvedev, "Continuous least-squares observers with applications," IEEE Transactions on Automatic Control, vol. 41, pp. 1530-1536, Oct. 1996.

[7] R. S. Bucy, "The Riccati equation and its bounds," Journal of Computer and System Sciences, vol. 6, 1972.

[8] M. Stocks and A. Medvedev, "Idealized two-axis models of faulty induction machines," CSEE / Control Engineering Group, Tech. Rep. ISSN 1402-1528 / ISRN LTU-FR-05/05-SE / NR 2005:05, 2005.

[9] M. Stocks, F. Rodyukov, and A. Medvedev, "Idealized two-axis model of induction machines under stator fault," in 1st IEEE Conference on Industrial Electronics and Applications ICIEA, Singapore, 2006.

[10] — "Idealized two-axis model of induction machines under rotor fault," in 1st IEEE Conference on Industrial Electronics and Applications ICIEA, Singapore, 2006.

[11] M. Stocks and A. Medvedev, "On observability and controllability of faulty induction machines," in 25th American Control Conference ACC, Minneapolis, USA, 2006. 